\title{
Analysis of clinical utilization of ring applicator for combined intracavitary/interstitial image-guided brachytherapy treatment in Chinese patients with locally advanced cervical cancer
}

\author{
Zhipeng Zhao, MS*, Ning Zhang, MS*, Ying Liu, BS*, Ning Wu, MD*, Zhuang Mao, BS, Wei Yang, BS, \\ Guanghui Cheng, MD, PhD \\ Department of Radiation Oncology, China-Japan Union Hospital of Jilin University, Changchun, China \\ *Zhipeng Zhao, Ning Zhang, Ying Liu, Ning Wu contributed equally to this work.
}

\begin{abstract}
Purpose: To analyze the results of clinical utilization of ring applicator for combined intracavitary (IC)/interstitial (IS) image-guided brachytherapy (BT) technique in locally advanced cervical cancer (LACC), and to explore the application conditions of IC/IS BT approach with this applicator for Chinese patients.

Material and methods: IC/IS BT was performed in total of 95 LACC patients, who already received external beam radiotherapy. In forty-three of these patients, utilization of ring applicator in brachytherapy was done, including IC/ IS BT approach with ring applicator for 65 fractions. Clinically optimized IC/IS BT plan with ring applicator (IC/IS treatment) and additionally generated optimized plan without needle use (IC research) were designed, respectively. Dosage differences in target regions and organs at risk (OARs) were analyzed between both the plans. Four planes were selected in the longitudinal direction of uterine cervix and divided the $360^{\circ}$ area around the tandem into eight equal sectors. Tumor target features and prescription dose distributions on different planes were described.

Results: The usage rate of ring applicator with most suitable ring diameter of $26 \mathrm{~mm}$ was $45.3 \%$ in Chinese cervical cancer patients. The high-risk clinical target volume (HR-CTV) $\mathrm{D}_{90} / \mathrm{D}_{98}$ and intermediate-risk CTV (IR-CTV) $\mathrm{D}_{90} /$ $\mathrm{D}_{98}$ in IC/IS treatment were significantly higher than IC research. As compared with IC research plan, the $\mathrm{D}_{2.0} \mathrm{~cm}^{3}$ of bladder and rectum in IC/IS treatment were significantly lower $(p<0.05)$. There was no significant difference in $\mathrm{D}_{2.0 \mathrm{~cm}^{3}}$ of sigmoid and small bowel between two plans. When the target volume was larger than $36 \mathrm{~cm}^{3}$, IC alone had an inadequate coverage of target.

Conclusions: Nearly half of Chinese patients with LACC could be treated with the ring applicator. The application conditions of IC/IS BT approach with ring applicator for Chinese patients were achieved. Combined IC/IS image-guided BT based on magnetic resonance imaging (MRI) planning is clinically feasible in Chinese patients.

Key words: cervical cancer, intracavitary, interstitial, brachytherapy, ring applicator.

\section{Purpose}

The standard treatment for locally advanced cervical cancer includes cisplatin-based concurrent chemo-radiotherapy, followed by brachytherapy. For brachytherapy treatment, different techniques such as intracavitary brachytherapy (ICBT), interstitial brachytherapy (ISBT), or hybrid intracavitary/interstitial brachytherapy (IC/ IS BT) are being increasingly applied for cervical cancer. ICBT has been previously described in the literature, and with this technique, excellent tumor control can be expected for small size tumors [1]. The American

Brachytherapy Society recommendations for high-doserate (HDR) brachytherapy for cervical carcinoma revealed that the eligibility criteria for undergoing ISBT were bulky lesions, narrow vagina, inability to enter cervical os, more than proximal third of parametrium extension, and lower vaginal extension [2]. In order to produce better target coverage while optimally sparing organs at risk (OARs), the combined IC/IS compatible computed tomography/magnetic resonance imaging (CT/MRI) applicator has a remarkable contribution. An analysis from the RetroEMBRACE study found that us-
\end{abstract}


ing combined IC/IS brachytherapy improves therapeutic ratio and local control in large tumors without adding treatment-related late morbidity [3]. Although the ring applicator is one of the most widely used applicators for IC/IS technique in the world, there are only few departments to implement IC/IS BT for cervical cancer using ring applicator, with less experience in China. However, the clinical feasibility of this applicator on a larger Chinese group of patients with cervical cancer have yet to be demonstrated. The aim of this present study was to investigate the preliminary results of clinical use and to analyze the indication of ring applicator in the treatment of Chinese patients with locally advanced cervical cancer.

\section{Material and methods}

\section{Patients}

A total of 95 patients with cervical squamous cell cancer (aged, $49.80 \pm 9.65$ years; Karnofsky Performance Score $[\mathrm{KPS}] \geq 70$ ) were enrolled into the study. These subjects were all Chinese of Han origin, inhabiting the Northeast area of China. The patients were admitted to our hospital between November 2014 and October 2016 Initial locoregional staging included a clinical evaluation with stage IB2 (9 patients), stage IIA2 (23 patients), stage IIIA (30 patients), stage IIIB (22 patients), and stage IVA (11 patients) was performed by a well-trained gynecologic surgeon and radiation oncologist according to the 2009 Federation International of Gynecology and Obstetrics (FIGO) classification.

All patients received pelvic external beam radiotherapy (EBRT) of $45 \mathrm{~Gy}$, with three-dimensional conformal radiotherapy (3D CRT) technique (18 patients) or intensity-modulated radiotherapy (IMRT) technique (77 patients) and 4 fractions of 3D IGBT (once a week) with 7 Gy of prescribed dose (PD). Brachytherapy was initiated at the $5^{\text {th }}$ week into EBRT (nearly 36-40 Gy). Then, 4 fractions of 3D IGBT were performed once a week. In addition, we applied 2 fractions of brachytherapy at the $8^{\text {th }}$ week in certain cases in order to control the total treatment time within 8 weeks. Chemotherapy was given during EBRT, with intravenous cisplatin $40 \mathrm{mg} / \mathrm{m}^{2}$ once a week, for 5-6 weeks in all patients.

\section{Equipment and applicator}

Micro-Selectron HDR Iridium-192 ( $\left.{ }^{192} \mathrm{Ir}\right)$ brachytherapy (Nucletron, an Elekta company, Elekta AB, Stockholm, Sweden), Oncentra v4.3, Nucletron (Veenendaal, The Netherlands), and 3.0T magnetic resonance imaging (Siemens, Erlangen, Germany) were used in the study. Interstitial ring CT/MRI applicator set (Nucletron, an Elekta company, Elekta AB, Stockholm, Sweden) is a gynecological applicator for brachytherapy procedures. This applicator is available in different diameters $(26,30$, and $34 \mathrm{~mm}$ ). Guide holes in the ring tube allow placement of MRI-compatible IS ProGuide needles using the ring tube as a needle template. A maximum of 9 ProGuide needles can be placed.

\section{Imaging, contouring, treatment planning, and dose reporting}

In each patient, an assessment of tumor position by MRI examination and clinical gynecological examination was implemented before first brachytherapy session. BT procedure with ultrasound-guided insertion of ring applicator under general anesthesia was performed. During the operation, the implant depths was adjusted according to the results of ultrasound guidance. After insertion, the needles were locked, and the rectal dose monitor was placed in the rectum. In the subsequent treatments, appropriate applicator should be chosen according to tumor shrinking condition.

Magnetic resonance images of every brachytherapy fraction were obtained by 3.0 T MRI scanner. Images were acquired according to our institutional protocol, which includes axial, sagittal, and coronal T2-weighted turbo spin-echo images, with $4.5 \mathrm{~mm}$ slice thickness and a balanced steady-state free precession scan with a slice thickness of $1.5 \mathrm{~mm}$ for applicator reconstruction purposes.

Based on the recommendations of the Gynecologic Groupe European de Curietherapie and the European Society for Therapeutic Radiology Oncology (GEC-ESTRO), the target regions and OARs in patients were contoured according to MRI images.

The target regions included high-risk clinical target volume (HR-CTV) and intermediate-risk clinical target volume (IR-CTV), and OARs involved bladder, rectum, sigmoid, and small bowel. Target volume delineation was based on the MRI image before and after external beam radiation.

On the basis of MRI images, the 3D IC/IS BT plan was optimized for HR-CTV $\mathrm{D}_{90} / \mathrm{D}_{98}$ (minimum doses delivered to $90 \%$ and $98 \%$ of the target volume) and OAR $\mathrm{D}_{2.0 \mathrm{~cm}^{3}}$ (minimum doses to $2 \mathrm{~cm}^{3}$ volumes of the OAR that receive the maximum dose). Dose calculation and reporting were based on the total (EBRT + BT) biologically equivalent dose in $2 \mathrm{~Gy}$ fractions $\left(\mathrm{EQD}_{2}\right)$. Linear quadratic (LQ) model for radiation damage repair was used with $\alpha / \beta=10$ Gy for the tumor target and $\alpha / \beta=3$ Gy for the OARs. The EQD ${ }_{2}$ of EBRT and BT was then added to evaluate the optimized plan regarding the dose-volume-histogram (DVH) constraints. The goal of combined EBRT and BT was set to achieve HR-CTV $D_{90} \geq 85 \mathrm{~Gy}$, IR-CTV $\mathrm{D}_{98} \geq 60 \mathrm{~Gy}$, bladder $\mathrm{D}_{2.0 \mathrm{~cm}^{3}} \leq 80 \mathrm{~Gy}$, and rectum/ sigmoid/small bowel $\mathrm{D}_{2.0 \mathrm{~cm}^{3}} \leq 70 \mathrm{~Gy}$. The DVH and the reference doses were used to design the treatment plan.

\section{Study analyses and experimental design}

Patients enrolled in this study who were treated with combined IC/IS BT with the ring applicator were defined as IC/IS treatment group. Each fractional treatment plan of IC/IS treatment group generated a simulation plan by changing the source dwell times of the tandem only, leaving the interstitial part aside, and it was defined as IC research group. This optimized IC research plan was not used clinically. The aim dose of target and the limit dose of OAR in IC research plan were the same as the criterion of IC/IS treatment group. 

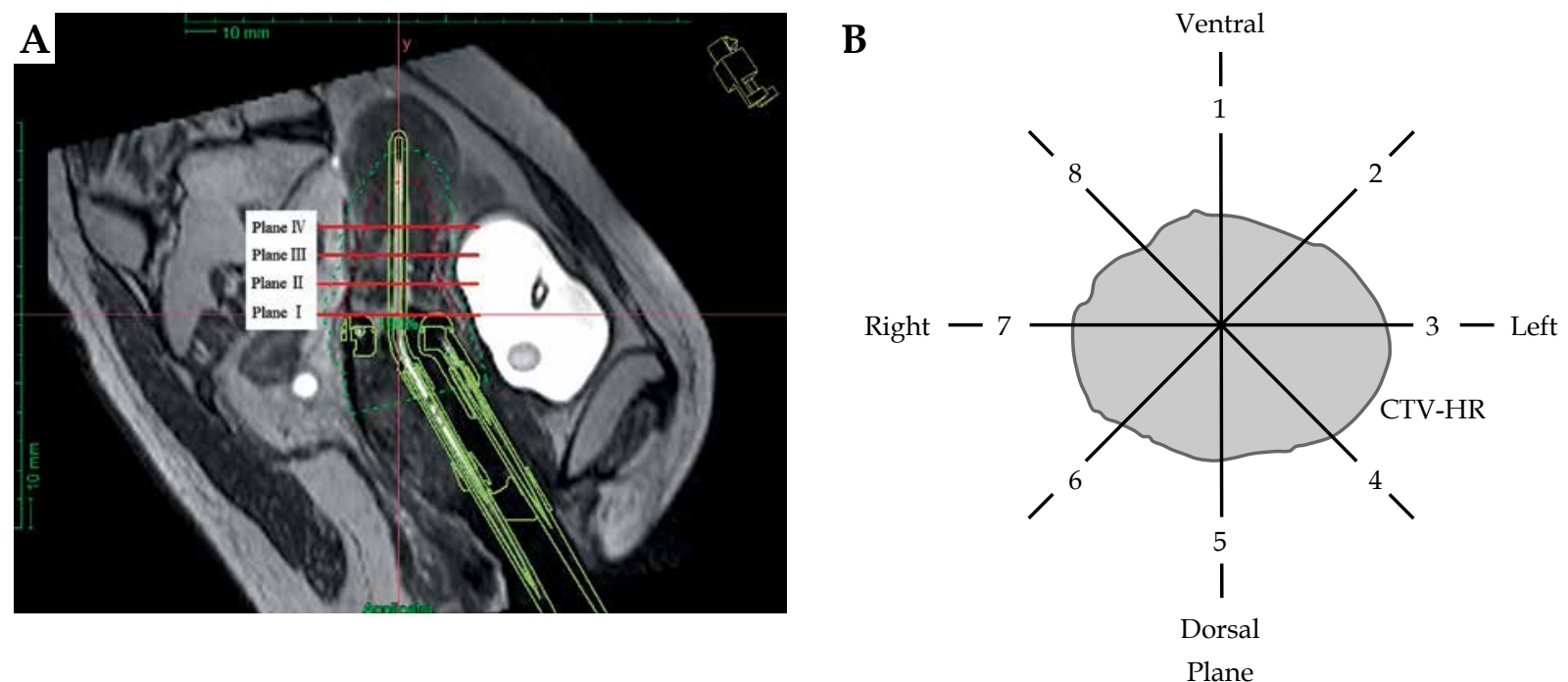

Fig. 1. Distribution of 4 planes and 8 directions. A) The 4 planes in sagittal planes. Plane I at the level of ring tube surface; Plane II at the parallel layer of $1 \mathrm{~cm}$ above ring tube surface; Plane III at the parallel layer of $2 \mathrm{~cm}$ above ring tube surface (ICRU point A); Plane IV at the parallel layer of $1 \mathrm{~cm}$ above plane III ( $3 \mathrm{~cm}$ above ring tube surface); B) The 8 directions in each plane

Each needle application was evaluated, and the number of needles, positions through the ring, and insertion depths were registered. These needle choices were compared with the location of HR-CTV. This location was determined in para-transverse planes (perpendicularly to the tandem). A total of 4 parallel planes were observed: plane I at the level of ring tube surface, plane II at the parallel layer of $1 \mathrm{~cm}$ above ring tube surface, plane III at the parallel layer of $2 \mathrm{~cm}$ above ring tube surface (ICRU point A), and plane IV at the parallel layer of $1 \mathrm{~cm}$ above plane III ( $3 \mathrm{~cm}$ above ring tube surface). We divided the $360^{\circ}$ area around the tandem into eight equal sectors. The dividing line from 1 to 8 in clockwise direction was numbered and the first line at $0 \mathrm{o}^{\prime}$ clock direction was marked (Figure 1).

We evaluated 65 fractions of IC/IS treatment group plans and the same fractions of IC research plans with the following criteria: for each direction of every plane analyzed, if the area of the target volume was covered by the prescription isodose curve, it was defined as qualified; if not, it was interpreted as unqualified. Therefore, we obtained the "qualified" ratio in each direction of every plane and in this way, the "prescription dose coverage rate" in each plane and direction was analyzed.

The HR-CTV $D_{90} / D_{98}$ and IR-CTV $D_{90} / D_{98}$ of target were calculated in the IC/IS treatment and IC research plans, respectively. Moreover, $\mathrm{D}_{2.0 \mathrm{~cm}^{3}}$ of OARs (bladder, rectum, sigmoid, and small bowel) were compared between the two treatment regimens. Tumor target features and prescription dose distributions on 4 planes were described.

\section{Data processing and statistical analysis}

Data were presented as mean \pm standard deviation. The comparison of dosimetric differences between two groups was done using pair $t$-test. Statistical significance was set at $p<0.05$ at two-sided level. The linear regression model was used for the HR-CTV $\mathrm{D}_{90}$ and target vol- ume analysis. All statistical analysis was performed using SPSS version 17.0 (SPSS, Chicago, IL, USA).

\section{Results}

\section{Treatment outcome}

In forty-three of 95 patients with locally advanced cervical cancer, the application of ring applicator was performed. The ring applicator usage rate was nearly $45.26 \%$. Forty-three cases were treated with 3D IGBT in 172 fractions, including implementation of IC/IS IGBT approach in 65 fractions.

The mean volume of HR-CTV was $48.2 \pm 27.2 \mathrm{~cm}^{3}$, and the mean volume of IR-CTV was $97.4 \pm 59.3 \mathrm{~cm}^{3}$.

\section{Dosimetric comparison of target exposure and OARs between the two plans}

Table 1 presents the dose parameters of target and OARs with ring applicator in the two investigated plans. The HR-CTV $\mathrm{D}_{90} / \mathrm{D}_{98}$ and IR-CTV $\mathrm{D}_{90} / \mathrm{D}_{98}$ in IC/IS treatment group were significantly higher than those in IC research group $(p<0.05)$. As compared with IC research plans, the $\mathrm{D}_{2.0} \mathrm{~cm}^{3}$ of bladder and rectum in IC/IS treatment plans were significantly lower $(p<0.05)$. There was no significant difference in the $\mathrm{D}_{2.0} \mathrm{~cm}^{3}$ of sigmoid and small bowel in the two plans. The total $\mathrm{EQD}_{2}$ dose of 43 patients treated with combined IC/IS BT was shown in Table 2.

\section{Needles implantation}

The total of 43 patients were treated by combined intracavitary/interstitial brachytherapy in 65 fractions. The total number of needles was 277 , and the average number of needles used was 4.3 for each patient, with the average depth of $4.1 \mathrm{~cm}$. Hole distributions in the ring applicator in our research are shown in Figure 2. The situation of each hole is shown in Table 3. 
Table 1. Comparison of dose parameters of target and OARs with ring applicator in two plans

\begin{tabular}{|c|c|c|c|c|c|}
\hline & & IC/IS treatment & IC research & $t$ & $P$-value \\
\hline \multirow[t]{2}{*}{ HR-CTV } & $D_{90}$ & $7.94 \pm 0.65$ & $6.98 \pm 0.75$ & 8.48 & $<0.001$ \\
\hline & $\mathrm{D}_{98}$ & $6.66 \pm 0.75$ & $5.85 \pm 0.66$ & 7.27 & $<0.001$ \\
\hline \multirow[t]{2}{*}{ IR-CTV } & $D_{90}$ & $4.86 \pm 0.53$ & $4.37 \pm 0.63$ & 7.07 & $<0.001$ \\
\hline & $D_{98}$ & $3.75 \pm 0.58$ & $3.34 \pm 0.69$ & 3.99 & $<0.001$ \\
\hline Bladder & $\mathrm{D}_{2.0 \mathrm{~cm} 3}$ & $5.18 \pm 0.66$ & $5.61 \pm 0.50$ & -6.71 & $<0.001$ \\
\hline Rectum & $\mathrm{D}_{2.0 \mathrm{~cm} 3}$ & $3.83 \pm 0.60$ & $4.05 \pm 0.49$ & -3.05 & 0.005 \\
\hline Sigmoid & $\mathrm{D}_{2.0 \mathrm{~cm} 3}$ & $3.56 \pm 0.90$ & $3.48 \pm 0.93$ & 1.76 & 0.091 \\
\hline Small bowel & $\mathrm{D}_{2.0 \mathrm{~cm} 3}$ & $2.68 \pm 1.32$ & $2.72 \pm 1.36$ & -1.32 & 0.199 \\
\hline
\end{tabular}

Table 2. Dose-volume histogram parameters in 43 patients treated with combined IC/IS BT

\begin{tabular}{lc} 
Parameters & $\begin{array}{c}\left.\mathrm{EQD}_{2} \text { dose (EBRT }+\mathrm{BT}\right) \\
\text { Mean } \pm \mathrm{SD}(\mathrm{Gy})\end{array}$ \\
\hline HR-CTV $\mathrm{D}_{90}$ & $90.33 \pm 9.20$ \\
\hline IR-CTV D & $67.89 \pm 3.59$ \\
\hline HR-CTV D & $80.17 \pm 7.45$ \\
\hline IR-CTV D & $62.15 \pm 4.11$ \\
\hline Bladder $\mathrm{D}_{2.0 \mathrm{~cm} 3}$ & $76.53 \pm 6.49$ \\
\hline Rectum $\mathrm{D}_{2.0 \mathrm{~cm} 3}$ & $64.25 \pm 6.76$ \\
\hline Sigmoid $\mathrm{D}_{2.0 \mathrm{~cm} 3}$ & $61.79 \pm 8.01$ \\
\hline Small bowel $D_{2.0 \mathrm{~cm} 3}$ & $60.15 \pm 6.80$
\end{tabular}

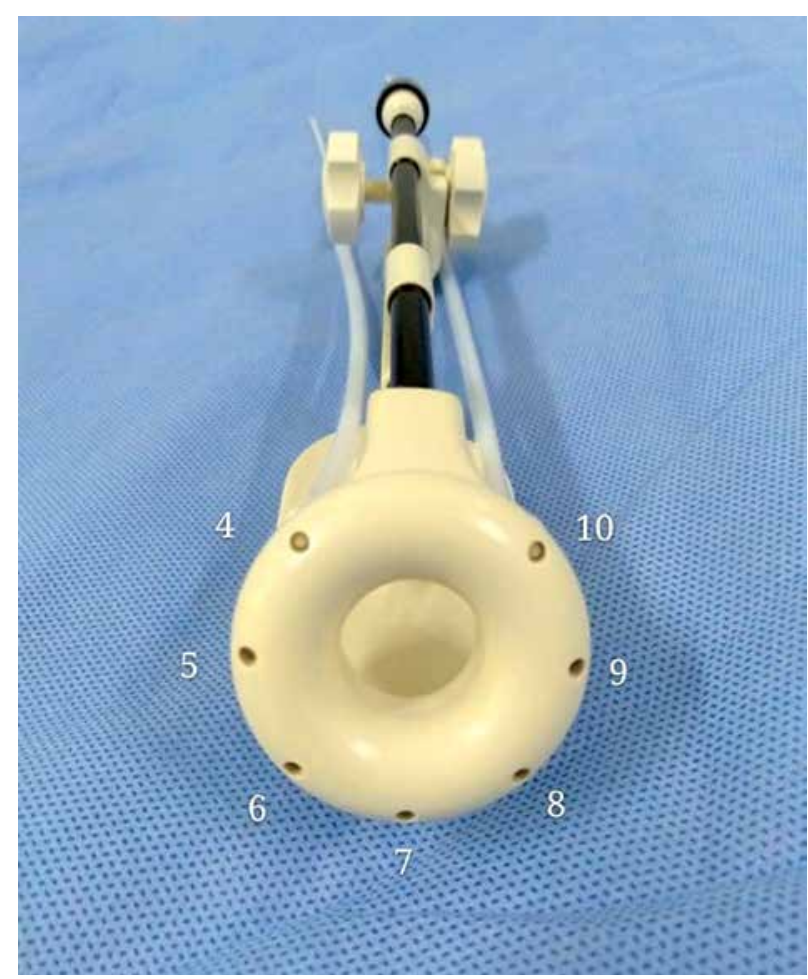

Fig. 2. Distribution of holes in the ring applicator

\section{Regression fitting of power function for target volume and $H R-C T V D_{90}$}

Regression fitting of power function with linear regression model as well as the relationship between target volume and HR-CTV $D_{90}$ under the two plans (Figure 3) was analyzed. According to the $\mathrm{EQD}_{2}$ value of LQ mod$\mathrm{el}$, when the $\mathrm{EQD}_{2}$ of HR-CTV $\mathrm{D}_{90}$ was $85 \mathrm{~Gy}$ in terms of four equivalent calculations, the physical dose of HRCTV $D_{90}$ was $7.13 \mathrm{~Gy}$. When HR-CTV $\mathrm{D}_{90}$ dose value was $7.13 \mathrm{~Gy}$, the target volume was $36 \mathrm{~cm}^{3}$ in ICBT curve. The result of regression curve analysis showed that it might be difficult for IC BT alone to meet the dose requirements of tumor target when its volume was equal to or greater than $36 \mathrm{~cm}^{3}$.

\section{Prescription dose coverage on different planes}

The prescription dose coverage rate in IC/IS treatment plans was $93.2 \%$, which was significantly higher than that in IC research $(69.7 \%)$. In IC research plans, the prescription dose could cover the target volume at 8 directions on plane I; the location of less than $60 \%$ of the prescription dose coverage were centered in the direction $3(48.1 \%)$, $4(44.4 \%), 6(51.8 \%)$, and $7(44.4 \%)$ on plane I, the maximum distance between prescription dose and tandem was $20 \mathrm{~mm}$ at these four directions; the location of less than $60 \%$ of prescription dose coverage were centered in the direction $3(44.4 \%), 4(48.1 \%), 6(44.4 \%)$, and 7 (51.8\%)

Table 3. Total number of implanted needles and average insertion depth in 65 cases treated with ring applicator

Needle channel Number of needles Average depth $(\mathrm{cm})$

\begin{tabular}{lll}
\hline 4 & 24 & 4.0 \\
\hline 5 & 58 & 4.2 \\
\hline 6 & 55 & 4.3 \\
\hline 7 & 17 & 2.9 \\
\hline 8 & 55 & 4.2 \\
\hline 9 & 51 & 4.3 \\
\hline 10 & 17 & 4.0
\end{tabular}




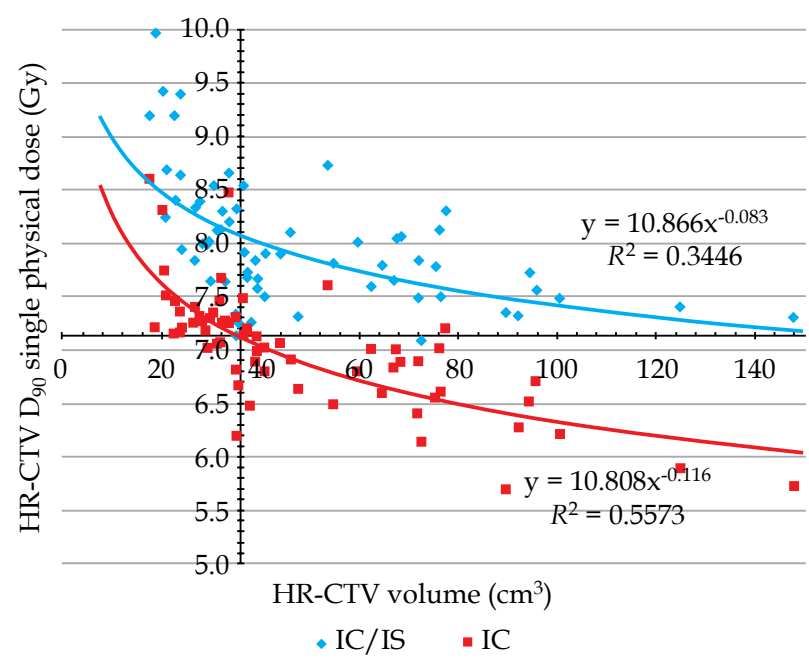

Fig. 3. Regression fitting of power function for target volume and HR-CTV $\mathrm{D}_{90}$ Blue curve: IC/ISBT fitting curve, red curve: ICBT fitting curve

According to the $E Q D_{2}$ value of the $L Q$ model, when the $E Q D_{2}$ of $H R$-CTV $D$ owas 85 Gy in terms of four equivalent calculations, the physical dose of HR-CTV $D_{90}$ was 7.13 Gy. When HR-CTV $D_{90}$ dose value was $7.13 \mathrm{~Gy}$, the target volume was $36 \mathrm{~cm}^{3}$ in red curve

on plane III; the maximum distance between prescription dose and tandem was $17.8 \mathrm{~mm}$ at these four directions; the location of less than $60 \%$ of prescription dose coverage were centered in the direction 6 (44.4\%) and 7 (51.8\%) on plane IV, the maximum distance between prescription dose and tandem was $17 \mathrm{~mm}$ at these two directions (Figure 4).

\section{Discussion}

The combined IC/IS BT can consider high-dose radiation and conformal dose distribution to target in brachytherapy procedure for locally advanced cervical cancer. The standardized applicators for IC/IS BT includes Utrecht applicator (tandem + ovoid + needles) and ring applicator (tandem + ring + needles). Because needles of ring applicator are parallel to tandem, it is more beneficial to anteversion of uterus. The interstitial ring CT/MR applicator set was introduced to China with minimal clinical practice experience. We analyzed the dosimetric comparisons of this ring applicator in order to provide a reliable reference for its clinical practice.

Only 43 patients could tolerate the ring applicator, which was limited to the minimum size (diameter with $26 \mathrm{~mm}$ ), and the usage rate of this applicator for combined IC/IS BT treatments was $45.26 \%$. In our study, we found that the ring applicator was applied to patients with anteversion of uterus or higher vaginal stretch conditions.

According to dosimetric comparison of the two plans, the target dose in IC/IS treatment plans was significantly higher than that in IC research plans. Moreover, IC/IS treatment plans could reduce the $\mathrm{D}_{2.0 \mathrm{~cm}^{3}}$ of rectum and bladder significantly, as compared with IC research plans. There was no significant difference in the dose of the sigmoid and small bowel in the two plans. This result was consistent with Fokdal et al. [3] that combined IC/IS BT to deliver significantly higher dose to the target area without increasing the dose to OARs.

In this study, the highest number of needles were implanted through the ring applicator at lateral and latero-posterior hole (Table 3). Similar results were found in previous studies $[4,5]$ using Utrecht applicator, and another study performed with tandem/ring applicator (GammaMed, Varian).

The dose constraint of rectum was more of rigorous standard in this study, since the higher increase of D2.0 $\mathrm{cm}^{3}$ of rectum, the higher incidence rate of side effects. Dose effect relationships have been demonstrated for rectal morbidity [6,7]. Georg et al. suggested that $\mathrm{D}_{2.0 \mathrm{~cm}^{3}}$ of rectum should be lower than $75 \mathrm{~Gy}$ [7], and when rectum $\mathrm{D}_{2.0 \mathrm{~cm}^{3}}$ dose further descended to $65 \mathrm{~Gy}$, the incidence of side effects was noted at below $10 \%$ [8]. When the rectum received an appropriate dose (60-70 Gy), only mild side effects occurred and included mainly fibrosis, telangiectasia, or inflammation [9]. The incidence of G2-G4 side effects was $8.6 \%$ in 3 years follow-up, when the $\mathrm{D}_{2.0 \mathrm{~cm}^{3}}$ of rectum was between 60 to $65 \mathrm{~Gy}$, which was the $1 / 3$ of incidence when the rectum $D_{2.0} \mathrm{~cm}^{3}$ was up to $75 \mathrm{~Gy}$ [7]. If the $\mathrm{D}_{2 \mathrm{~cm}^{3}}$ of rectum was more than $75 \mathrm{~Gy}$, the risk of rectal fistula was nearly $12.5 \%$. Therefore, the appropriate rectal $\mathrm{D}_{2.0 \mathrm{~cm}^{3}}$ dose limit for this study was 70 Gy.

Mazeron et al. found that the incidence of G2-G4 side effects in rectum and bladder was related to the $\mathrm{D}_{2.0} \mathrm{~cm}^{3}$. When $\mathrm{D}_{2.0} \mathrm{~cm}^{3}$ was less than $81.4 \mathrm{~Gy}$, the incidence of adverse reaction of bladder $(30 \%)$ was higher than that of the rectum [10]. As the radiation dose increased, the sensitivity of the bladder to the dose response decreased. In this study, the restriction dose of bladder was set as $80 \mathrm{~Gy}$. If the HR-CTV $\mathrm{D}_{90}$ exceeded $87 \mathrm{~Gy}\left(\mathrm{EQD}_{2}\right)$, the 3 -year local control results could be better than 95\% [11]. In order to improve the local control, this study required the HR-CTV $D_{90}$ to reach 85 Gy or more, with the HR$\mathrm{CTV} \mathrm{D}_{90}$ requirement appropriately increased, when there are high-risk factors to consider. For example, in case of stage III-IV cervical cancer patient, target volume $>30 \mathrm{~cm}^{3}$, treatment time $>55$ days, and tumor diameter $>5 \mathrm{~cm}$ at diagnosis, etc., the HR-CTV $\mathrm{D}_{90}$ needs to be increased to $97,92,105$, and 92 Gy, respectively [12].

In the present study, the physical dose of HR-CTV $\mathrm{D}_{90}$ obtained by the two plans was fitted to the regression curve of the target volume. According to $\mathrm{EQD}_{2}$ value in LQ model, when the $\mathrm{EQD}_{2}$ of $\mathrm{HR}-\mathrm{CTV} \mathrm{D}_{90}$ was $85 \mathrm{~Gy}$ in terms of four equivalent calculations, the physical dose of HR-CTV $D_{90}$ was equal to $7.13 \mathrm{~Gy}$. The result of regression curve analysis showed that it might be difficult for IC BT alone to meet the dose requirements of tumor target when its volume was equal to or greater than $36 \mathrm{~cm}^{3}$ in Chinese patients with locally advanced cervical cancer. Yoshida et al. used a cube as a model to investigate the HR-CTV volume threshold of IC BT alone [13]. It was considered that HR-CTV less than $4 \times 3 \times 3 \mathrm{~cm}^{3}$ (equivalent to $36 \mathrm{~cm}^{3}$ ) required image-guided IC BT and recommended IC/IS BT or IS BT for HR-CTV larger than this volume. This result was calculated using a cube as a model. When the model was converted to an ovoid for 

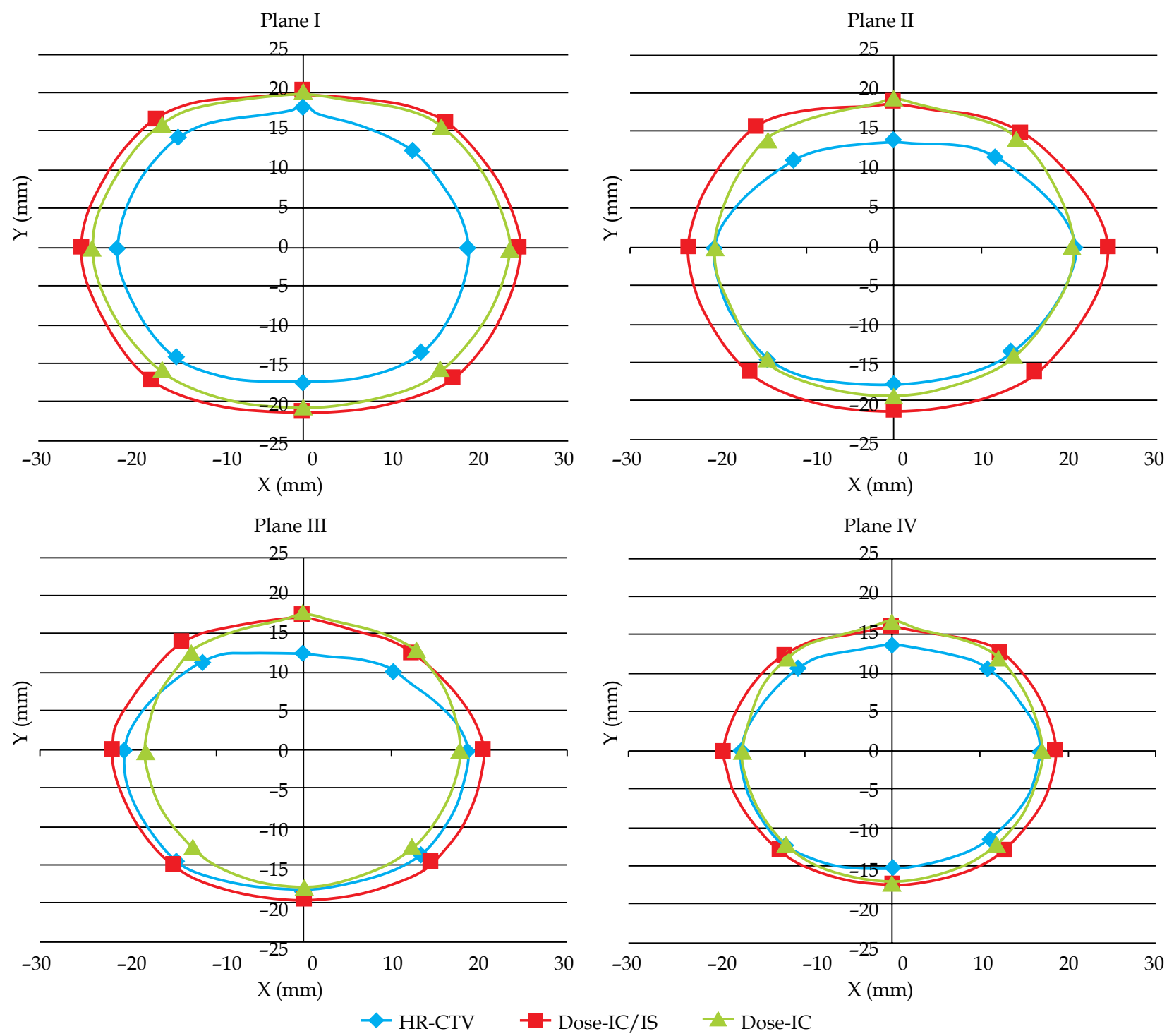

Fig. 4. The relationship between HR-CTV and PD coverage on different planes in ICIS treatment and IC research plans

The blue : the average distance from HR-CTV to the tandem. The red : the average distance from prescribed dose isodose curve of ICIS treatment plans to the tandem. The green $\Delta$ : the average distance from prescribed dose isodose curve of IC research plans to the tandem

volume calculation, this critical volume was only $18.8 \mathrm{~cm}^{3}$, which was smaller than the results of this study. The reasons for this difference may be as follows. First, the HR-CTV in the Yoshida et al.'s study was obtained by the volume calculation formula and might have some differences from the direct measurement of clinical target volume in this study. Second, the shape of HR-CTV was different in the two studies. The HR-CTV studied by Yoshida et al. was a fully symmetrically distributed cube. However, the HR-CTV in this study was the actual target region, and the full symmetry of the shape was difficult to achieve. Third, the Yoshida et al.'s study selected fewer models with a total of 8 different HR-CTV volumes $(8,12$, $27,36,64,80,96$, and $\left.112 \mathrm{~cm}^{3}\right)$. However, the number of patients enrolled in this research was large, and the difference in target volume was insignificant. The estimated value was fitted by the regression curve relationship between target volume and HR-CTV. This is also the main reason for the large difference between the two data. In the study of Yoshida et al., the tandem just passed the HR-CTV center, which was rare in actual clinical situation. Anderson et al. suggested that the conventional IC BT alone could meet the clinical requirements of HR-CTV $\mathrm{D}_{90}$ for a $<40 \mathrm{~cm}^{3} \mathrm{HR}-\mathrm{CTV}$. When HR-CTV $>40 \mathrm{~cm}^{3}$, IC BT combined with image guidance was required. When the volume of HR-CTV was further increased (median, $74 \mathrm{~cm}^{3}$ ), the target coverage evaluated by HR- CTV $\mathrm{D}_{90}$ in image-guided IC BT could reach only $80 \pm 16 \%$ (PD\%), and IC/IS BT was recommended [14]. The result of volume threshold reported by Anderson et al. was greater than that estimated in our study, which might be related to higher doses of OAR in their research.

In this study, the target area coverage of IC/IS BT was much higher than that of IC BT alone (93.2\% vs. $69.7 \%)$, which was related to better conformation of PD curve to the coverage of target in IC/ISBT insertion needles. It was 
found that the location of coverage rate ratio less than $60 \%$ in IC research plans were concentrated in direction $3,4,6$, and 7 on the plane II and III; direction 6 and 7 on the plane IV. In addition, on the III and IV planes, the PD curve formed by IC BT created approximately a regular circle. Plane I in this study was the parallel layer for upper surface of the ring tube. In clinical applications, larger diameter of ring applicator should be selected, so that the shape of ring tube and the cervix front-end could be as consistent as possible. In this case, both IC BT and IC/IS BT could produce good dose coverage of tumor target on plane I. At planes II and III, IC BT alone could not fully cover the dorso-lateral part of HR-CTV, which was related to the distribution of target area. Coia et al. reported that the irregular shape of target volume and paracervical excessive invasion were the main reasons to unsatisfactory dose coverage and insufficient dose distribution [15]. In our study, plane IV was located at the parallel layer of $3 \mathrm{~cm}$ above ring tube surface, and the dose coverage of IC BT was better than that in planes II and III. The possible reason might be that the height of some HR-CTV was less than $3 \mathrm{~cm}$. It could be seen that there was a minimum dose contribution of the ring tube at the parallel layer of ICRU point A or above, and the radiation dose was likely to be insufficient in the direction of dorsal part of tumor target.

In the present study, when arbitrary directions in the lower quadrant (directions 3, 4, 6, and 7) of the plane II exceeded $20 \mathrm{~mm}$, and the arbitrary directions of planes III and IV exceeded 17.8 and $17 \mathrm{~mm}$, respectively, it was difficult to satisfy the coverage requirements of the target area by IC BT alone. However, Dimopoulos et al. evaluated their experience with tandem/ring/needles applicator for cervical cancer [16]. The IC/IS BT was selected on the condition that the unilateral tumor expanded more than $3.5 \mathrm{~cm}$ at the ring level, and/or more than $2.5 \mathrm{~cm}$ at ICRU point A level, and/or more than $2.2 \mathrm{~cm}$ at a distance of 3 to $4 \mathrm{~cm}$ from the upper ring surface. The differences between the anatomy and the ring applicator diameter applied might be the actual reasons for discrepancies in the above studies. In addition, the relatively strict dose limitation in our research was also an important factor.

\section{Conclusions}

The IC/IS BT technique with the ring applicator was chosen if IC BT alone was unable to produce sufficient coverage of target volume with the PD. This occurred in two clinical situations: 1 . if HR-CTV was more than $36 \mathrm{~cm}^{3}$, and 2. if the cervical dorsal tumor extension exceeded $20 \mathrm{~mm}$ at a distance $1 \mathrm{~cm}$ cranial to the upper ring surface, or unilateral tumor extension exceeded $17.8 \mathrm{~mm}$ at the level of ICRU point $\mathrm{A}$ and $17 \mathrm{~mm}$ at a distance $3 \mathrm{~cm}$ cranial to the upper ring surface. Combined IC/IS image-guided BT based on MRI treatment planning with the ring applicator in situ is clinically feasible in Chinese patients with locally advanced cervical cancer, which need a reasonable selection of indications. Our experience with 95 patients has helped us to create institutional guidelines. In the near future, we expect that IC/IS image-guided BT in combination with the ring applicator will make an important contribution to the treatment of cervical cancer in China.

\section{Acknowledgements}

We would like to thank the other staff of the Departments of Radiology, Anesthesiology, and Nursing staff for helping us in many ways during the completion of this paper.

\section{Funding}

This work was partially supported by grants from the National Natural Science Foundation of China (grant numbers 81201737, 31600679), the Project of Science and Technology Development Plan of Jilin Province (grant number 20200201524JC), the Project of Health and Family Planning Commission of Jilin Province (grant number 2014ZC054), the Bethune Special Research of Science and Technology Department of Jilin Province (grant number 20160101079JC), the Horizontal Project of Jilin University (grant numbers 2015373, 2016220101000686), the Jilin University Technical Services Research Foundation (grant number 2015YX154), the Jilin University Network Experiment Project (grant number VE2015081), the Jilin University Undergraduate Education Reform Research Project (grant number 2017XYB0800), the Jilin University Norman Bethune Medical Department Teaching Reform Research Project (grant number B2014B137), and the Project of Science and Technology Department of Jilin Province (grant number 20190303151SF).

\section{Disclosure}

The authors report no conflict of interest.

\section{References}

1. Hsu IC, Speight J, Hai J et al. A comparison between tandem and ovoids and interstital gynecologic template brachytherapy dosimetry using a hypothetical computer model. Int J Radiat Oncol Biol Phys 2002; 52: 538-543.

2. Viswanathan AN, Thomadsen B. American Brachytherapy Society consensus guidelines for locally advanced carcinoma of the cervix. Part I: General principles. Brachytherapy 2012; 11: 33-46.

3. Fokdal L, Sturdza A, Mazeron R et al. Image guided adaptive brachytherapy with combined intracavitary and interstitial technique improves the therapeutic ratio in locally advanced cervical cancer: Analysis from the retroEMBRACE study. Radiother Oncol 2016; 20: 434-440.

4. Nomden CN, De Leeuw AA, Moerland MA et al. Clinical use of the Utrecht applicator for combined intracavitary/interstitial brachytherapy treatment in locally advanced cervical cancer. Int J Radiat Oncol Biol Phys 2012; 82: 1424-1430.

5. Fokdal L, Tanderup K, Hokland SB et al. Clinical feasibility of combined intracavitary/interstitial brachytherapy in locally advanced cervical cancer employing MRI with a tandem/ring applicator in situ and virtual preplanning of the interstitial component. Radiother Oncol 2013; 107: 63-68.

6. Chopra S, Dora T, Engineer R et al. Late rectal toxicity after image-based high-dose-rate interstitial brachytherapy for postoperative recurrent and/or residual cervical cancers: EQD2 predictors for grade $\geq$ II toxicity. Brachytherapy 2015; 14: $881-888$ 
7. Georg P, Lang S, Dimopoulos JC et al. Dose-volume histogram parameters and late side effects in magnetic resonance image-guided adaptive cervical cancer brachytherapy. Int J Radiat Oncol Biol Phys 2011; 79: 356-362.

8. Mazeron R, Fokdal LU, Kirchheiner K et al. Dose-volume effect relationships for late rectal morbidity in patients treated with chemoradiation and MRI-guided adaptive brachytherapy for locally advanced cervical cancer: Results from the prospective multicenter EMBRACE study. Radiother Oncol 2016; 120: 412-419.

9. Pötter R, Haie-Meder C, Limbergen EV et al. Recommendations from gynaecological (GYN) GEC ESTRO working group (II): Concepts and terms in 3D image-based treatment planning in cervix cancer brachytherapy - 3D dose volume parameters and aspects of 3D image-based anatomy, radiation physics, radiobiology. Radiother Oncol 2006; 78: 67-77.

10. Mazeron R, Maroun P, Castelnau-Marchand P et al. Pulseddose rate image-guided adaptive brachytherapy in cervical cancer: Dose-volume effect relationships for the rectum and bladder. Radiother Oncol 2015; 116: 226-232.

11. Dimopoulos JC, Potter R, Lang S et al. Dose-effect relationship for local control of cervical cancer by magnetic resonance image-guided brachytherapy. Radiother Oncol 2009; 93: 311-315.

12. Mazeron R, Castelnau-Marchand P, Dumas I et al. Impact of treatment time and dose escalation on local control in locally advanced cervical cancer treated by chemoradiation and image-guided pulsed-dose rate adaptive brachytherapy. Radiother Oncol 2015; 114: 257-263.

13. Yoshida K, Yamazaki H, Kotsuma T et al. Simulation analysis of optimized brachytherapy for uterine cervical cancer: Can we select the best brachytherapy modality depending on tumor size? Brachytherapy 2016; 15: 57-64.

14. Anderson JW, Xia J, Flynn RT et al. High resolution (3 Tesla) MRI-guided conformal brachytherapy for cervical cancer: consequences of different high-risk CTV sizes. J Contemp Brachytherapy 2013; 2: 101-109.

15. Coia L, Won M, Lanciano R et al. The patterns of care outcome study for cancer of the uterine cervix. Results of the Second National Practice Survey. Cancer 1990; 66: 2451-2456.

16. Dimopoulos JCA, Kirisits C, Petric P et al. The Vienna applicator for combined intracavitary and interstitial brachytherapy of cervical cancer: Clinical feasibility and preliminary results. Int J Radiat Oncol Biol Phys 2006; 66: 83-90. 